\title{
Analysis of Power Law Non-Linearity in Solitonic Solutions Using Extended Hyperbolic Function Method
}

Naeem Ullah

The University of Lahore

Muhammad Imran Asjad

The University of Lahore

Taseer Muhammad

King Khalid University

Ali Akgül ( $\square$ aliakgul00727@gmail.com )

Siirt Universitesi https://orcid.org/0000-0001-9832-1424

\section{Research Article}

Keywords: New EHFM, Power law non-linearity, Optical solitons.

Posted Date: January 18th, 2022

DOI: https://doi.org/10.21203/rs.3.rs-1243415/v1

License: (c) (i) This work is licensed under a Creative Commons Attribution 4.0 International License. Read Full License 


\title{
Analysis of power law non-linearity in solitonic solutions using extended hyperbolic function method
}

\author{
Naeem Ullah $^{1}$, Muhammad Imran Asjad ${ }^{1}$, Taseer Muhammad ${ }^{2}$, Ali Akgül ${ }^{4, *}$ \\ ${ }^{1}$ Department of Mathematics, University of Management and Technology, Lahore, \\ 54770, Pakistan. \\ ${ }^{2}$ Department of Mathematics, College of Sciences, King Khalid University, Abha \\ 61413, Saudi Arabia. \\ ${ }^{3}$ Siirt University, Art and Science Faculty, Department of Mathematics, 56100 Siirt, \\ Turkey. \\ Corresponding Author: * aliakgul00727@gmail.com
}

\begin{abstract}
This paper retrieves the optical solitons to Biswas-Arshed equation (BAE) which is deliberated in the lack of self-phase modulation by applying the new extended hyperbolic function method (EHFM). Novel constructed solutions have the shape of bright, singular, periodic singular, and dark solitons. The achieved solutions contain key applications in engineering and physics. These solutions define the wave performance of the governing models, actually. The outcomes disclose that our scheme is very active and reliable. The acquired results are illustrated by $3-\mathrm{D}$ and $2-\mathrm{D}$ graphs to understand the real phenomena for such sort of non-linear models.
\end{abstract}

Keywords: New EHFM; Power law non-linearity; Optical solitons.

\section{Introduction}

Solitons have much importance in various areas of science and nature such as waves, geology, wave propagation, population ecology, fluid dynamics, computer science, biology, plasmas, heat, birefringent fibers, mechanics and optics. There are many mathematical models that efficiently demonstrate the properties of soliton transmission [1-36]. The key object for the presence of solitons in the optical fibers is to keep balance among GVD and nonlinearity. In special conditions, there may a circumstances arise which produce small nonlinearity and low GVD. Recently, Biswas and Arshed [37-42] estimated a precise creative clue to grip the circumstances where non-linearity and GVD are small which is prented in the model called BAE. This governing model can act as a feasible model to study the properties of solitons in crystals, PCF, and optical fibers. This article studies the BAE with power law via new EHFM [43-45] that lead to construct singular, bright, dark-bright and periodic-singular optical soliton solutions. 
The layouts of this paper are as. The governing model is described in section 2. Analysis of the proposed method new EHFM is presented in section 3. In section 4 the new EHFM is applied and section 5 consists the findings and discussions. Conclusions of this paper are posted in section 6 .

\section{Biswas-Arshed Equation}

The BAE with full non-linearity [37-42] is given by

$$
\iota u_{t}+a_{1} u_{x x}+a_{2} u_{x t}+\iota\left(b_{1} u_{x x x}+b_{2} u_{x x t}\right)=\iota\left[\phi\left(|u|^{2 n} u\right)_{x}+\sigma\left(|u|^{2 n}\right)_{x} u+\theta|u|^{2 n} u_{x}\right],
$$

$u(x, t)$ and $n$ are complex-valued function and full nonlinearity parameter respectively. On the left of the (1), $a_{1}$ and $a_{2}$ indicate the temporal evolution, coefficient of GVD and coefficient of STD respectively. Next, $b_{1}$ and $b_{2}$ are coefficients of 3OD and STD. $\theta$ and $\sigma$ represent the effect of self-steepening and non-linear dispersion in the absence of SPM. This effect of dispersion and nonlinearity provides required balance for solitons existence.

Assume that

$$
u(x, t)=P(\eta) e^{\iota \Psi(x, t)}
$$

where $P(\eta)$ indicate the amplitude and

$$
\eta=x-c t, \quad \Psi(x, t)=-k x+w t+\epsilon .
$$

where $c$ and $\Psi(x, t)$ are the velocity and the phase component. While $k, w$ are frequency and wave number, accordingly, while $\epsilon$ is phase constant of solitons.

Inserting (2), (3) in (1) yields:

The real part gives

$$
\begin{array}{r}
-\left(w+a_{1} k^{2}+b_{1} k^{3}-a_{2} w k-b_{2} w k^{2}\right) P+\left(a_{1}+3 b_{1} k-a_{2} c-2 b_{2} c k-b_{2} w\right) P^{\prime \prime} \\
=k(\theta+\phi) P^{2 n+1}
\end{array}
$$

while the imaginary part gives

$$
\begin{array}{r}
\left(-3 b_{1} k^{2}+b_{2} c k^{2}+2 b_{2} w k-2 a_{1} k-c+2 a_{2} c k+a_{2} w\right) P^{\prime}+\left(-b_{2} c+b_{1}\right) P^{\prime \prime \prime} \\
=[\theta+2 n \sigma+(2 n+1) \phi] P^{2 n} P^{\prime}
\end{array}
$$

Integrating (5) and taking the integration constant to be zero, we obtain

$$
\begin{array}{r}
\left(-3 b_{1} k^{2}+b_{2} c k^{2}+2 b_{2} w k-2 a_{1} k-c+2 a_{2} c k+a_{2} w\right) P+\left(-b_{2} c+b_{1}\right) P^{\prime \prime} \\
=[\theta+2 n \sigma+(2 n+1) \phi] P^{2 n} .
\end{array}
$$

To get the solutions, we use following substitution

$$
P=W^{1 / 2 n}
$$


Eqs. (5) and (6), this gives yields

$$
\begin{array}{r}
\left(a_{1}+3 b_{1} k-a_{2} c-b_{2} w-2 b_{2} c k\right)\left[(1-2 n)\left(W^{\prime}\right)^{2}+2 n W W^{\prime \prime}\right] \\
-4 n^{2}\left(w+a_{1} k^{2}+b_{1} k^{3}-a_{2} w k-b_{2} w k^{2}\right) W^{2}-4 n^{2} k(\theta+\phi) W^{3}=0 \\
(2 n+1)\left(b_{1}-b_{2} c\right)\left[(1-2 n)\left(W^{\prime}\right)^{2}+2 n W W^{\prime \prime}\right]-4 n^{2}[2 n \sigma+\theta+(2 n+1) \phi] W^{3} \\
+\left(-2 a_{1} k-3 b_{1} k^{2}+b_{2} c k^{2}+2 b_{2} w k+2 a_{2} c k-c+a_{2} w\right) 4 n^{2}(2 n+1)\left[(1-2 n)\left(W^{\prime}\right)^{2}\right. \\
\left.+2 n W W^{\prime \prime}\right] W^{2}=0 .
\end{array}
$$

As $W(\xi)$ satisfies (8) and (9), the constraint conditions are as follows

$$
\begin{gathered}
\quad \frac{\left(a_{1}-a_{2} c+3 b_{1} k-2 b_{2} c k-b_{2} w\right)}{(2 n+1)\left(b_{1}-b_{2} c\right)}=\frac{(\theta+\phi) k}{2 n \sigma+\theta+(2 n+1) \phi} \\
=\frac{-\left(w+a_{1} k^{2}+b_{1} k^{3}-a_{2} w k-b_{2} w k^{2}\right)}{(2 n+1)\left(b_{2} c k^{2}-3 b_{1} k^{2}+2 b_{2} w k-2 a_{1} k+2 a_{2} c k-c+a_{2} w\right)} .
\end{gathered}
$$

Now (8) is analyzed using extended hyperbolic function method.

Setting $A=b_{2} c k^{2}-3 b_{1} k^{2}+2 b_{2} w k-2 a_{1} k+2 a_{2} c k-c+a_{2} w$ and $B=w+a_{1} k^{2}-a_{2} w k+b_{1} k^{3}-b_{2} w k^{2}$, gives

$$
A\left[(1-2 n)\left(W^{\prime}\right)^{2}+2 n W W^{\prime \prime}\right]-4 n^{2} B W^{2}-4 n^{2} k(\theta+\phi) W^{3}=0 .
$$

\section{New EHFM}

Two phases of the new EHFM are

Form 1: Let (11) has the solution as

$$
W(\eta)=\sum_{i=0}^{N} F_{i} \Phi^{i}(\eta),
$$

where $F_{i}$ are constants and $\Phi(\eta)$ satisfies the auxiliary ODE as

$$
\frac{d \Phi}{d \eta}=\Phi \sqrt{\tau+\mu \Phi^{2}}, \tau, \mu \in R
$$

To find the number $N$, we use balancing rule on (11). Substituting (12) in (11) along with (13) produces a system of algebraic equations for $F_{i}(0 \leq i \leq N)$. A set of solutions is acquired by solving this system, that accepts (13) as

Set 1: If $\tau>0$ and $\mu>0$,

$$
\Phi(\eta)=-\sqrt{\frac{\tau}{\mu}} \operatorname{csch}\left(\sqrt{\tau}\left(\eta+\eta_{0}\right)\right) .
$$


Set 2: If $\tau<0$ and $\mu>0$,

$$
\Phi(\eta)=\sqrt{\frac{-\tau}{\mu}} \sec \left(\sqrt{-\tau}\left(\eta+\eta_{0}\right)\right) .
$$

Set 3: If $\tau>0$ and $\mu<0$,

$$
\Phi(\eta)=\sqrt{\frac{\tau}{-\mu}} \operatorname{sech}\left(\sqrt{\tau}\left(\eta+\eta_{0}\right)\right) .
$$

Set 4: If $\tau<0$ and $\mu>0$,

$$
\Phi(\eta)=\sqrt{\frac{-\tau}{\mu}} \csc \left(\sqrt{-\tau}\left(\eta+\eta_{0}\right)\right) .
$$

Set 5: If $\tau>0$ and $\mu=0$,

$$
\Phi(\eta)=\exp \left(\sqrt{\tau}\left(\eta+\eta_{0}\right)\right)
$$

Set 6: If $\tau<0$ and $\mu=0$,

$$
\Phi(\eta)=\cos \left(\sqrt{-\tau}\left(\eta+\eta_{0}\right)\right)+\iota \sin \left(\sqrt{-\tau}\left(\eta+\eta_{0}\right)\right) .
$$

Set 7: If $\tau=0$ and $\mu>0$,

$$
\Phi(\eta)= \pm \frac{1}{\left(\sqrt{\mu}\left(\eta+\eta_{0}\right)\right)} .
$$

Set 8: If $\tau=0$ and $\mu<0$,

$$
\Phi(\eta)= \pm \frac{\iota}{\left(\sqrt{-\mu}\left(\eta+\eta_{0}\right)\right)}
$$

Form 2: Adopting the same pattern as above, assume (12) satisfies the auxiliary ODE as follows

$$
\frac{d \Phi}{d \eta}=\tau+\mu \Phi^{2}, \tau, \mu \in R
$$

Substituting (12) into (11) with (22) yields value of $N$, provides a set equations. Assume (22) has the solutions as

Set 1: If $\tau \mu>0$,

$$
\Phi(\eta)=\operatorname{sn}(\tau) \sqrt{\frac{\tau}{\mu}} \tan \left(\sqrt{\tau \mu}\left(\eta+\eta_{0}\right)\right)
$$


Set 2: If $\tau \mu>0$,

$$
\Phi(\eta)=-s n(\tau) \sqrt{\frac{\tau}{\mu}} \cot \left(\sqrt{\tau \mu}\left(\eta+\eta_{0}\right)\right) .
$$

Set 3: If $\tau \mu<0$,

$$
\Phi(\eta)=\operatorname{sn}(\tau) \sqrt{\frac{\tau}{-\mu}} \tanh \left(\sqrt{-\tau \mu}\left(\eta+\eta_{0}\right)\right) .
$$

Set 4: If $\tau \mu<0$,

$$
\Phi(\eta)=\operatorname{sn}(\tau) \sqrt{\frac{\tau}{-\mu}} \operatorname{coth}\left(\sqrt{-\tau \mu}\left(\eta+\eta_{0}\right)\right) .
$$

Set 5: If $\tau=0$ and $\mu>0$,

$$
\Phi(\eta)=-\frac{1}{\mu\left(\eta+\eta_{0}\right)} .
$$

Set 6: If $\tau \in R$ and $\mu=0$,

$$
\Phi(\eta)=\tau\left(\eta+\eta_{0}\right) .
$$

Note: $s n$ is well-known sign function.

\section{Application of the new EHFM}

Form 1: In this section, we utilize the above said method to solve the BAE with power law non-linearity. Using balance principal in (11), yields $N=2$, so (12) converts to

$$
W(\eta)=F_{0}+F_{1} \Phi(\eta)+F_{2}(\Phi(\eta))^{2},
$$

where $F_{0}, F_{1}$ and $F_{2}$ are constants. Inserting (29) in (11) and attains a set of equations in $F_{0}, F_{1}, F_{2}, \tau$ and $\mu$ is obtained. On working the set of equations, we achieve

$$
\begin{gathered}
F_{0}=0, \quad F_{1}=0, \\
F_{2}=F_{2}, \quad \tau=-\frac{B n^{2}}{2 A n-2 n-A}, \\
\mu=-\frac{k n^{2}(\theta+\phi) F_{2}}{2 A n-3 n-A},
\end{gathered}
$$


Set 1: If $\tau>0$ and $\mu>0$,

$$
\begin{aligned}
u_{1}(x, t)=\left[F_{2}\left(-\sqrt{\frac{B(-3 n+A(-1+2 n))}{k(-2 n+A(-1+2 n))(\theta+\phi) F_{2}}} \operatorname{csch}\left(\sqrt{-\frac{B n^{2}}{2 A n-2 n-A}}\left(\eta+\eta_{0}\right)\right)\right)^{2}\right]^{1 / 2 n} \\
\times\left(e^{\iota \Psi(x, t)}\right) .
\end{aligned}
$$

Set 2: If $\tau<0$ and $\mu>0$,

$$
\begin{aligned}
u_{2}(x, t)=\left[F_{2}\left(\sqrt{-\frac{B(-3 n+A(-1+2 n))}{k(-2 n+A(-1+2 n))(\theta+\phi) F_{2}}} \sec \left(\sqrt{\frac{B n^{2}}{2 A n-2 n-A}}\left(\eta+\eta_{0}\right)\right)\right)^{2}\right]^{1 / 2 n} \\
\times\left(e^{\iota \Psi(x, t)}\right) .
\end{aligned}
$$

Set 3: If $\tau>0$ and $\mu<0$,

$$
\begin{aligned}
u_{3}(x, t)=\left[F_{2}\left(\sqrt{-\frac{B(-3 n+A(-1+2 n))}{k(-2 n+A(-1+2 n))(\theta+\phi) F_{2}}} \operatorname{sech}\left(\sqrt{-\frac{B n^{2}}{2 A n-2 n-A}}\left(\eta+\eta_{0}\right)\right)\right)^{2}\right]^{1 / 2 n} \\
\times\left(e^{\iota \Psi(x, t)}\right) .
\end{aligned}
$$

Set 4: If $\tau<0$ and $\mu>0$,

$$
\begin{aligned}
u_{4}(x, t)=\left[F_{2}\left(\sqrt{-\frac{B(-3 n+A(-1+2 n))}{k(-2 n+A(-1+2 n))(\theta+\phi) F_{2}}} \csc \left(\sqrt{\frac{B n^{2}}{2 A n-2 n-A}}\left(\eta+\eta_{0}\right)\right)\right)^{2}\right]^{1 / 2 n} \\
\times\left(e^{\iota \Psi(x, t)}\right) .
\end{aligned}
$$

Set 5: If $\tau>0$ and $\mu=0$,

$$
u_{5}(x, t)=\left[F_{2}\left(\exp \left(\sqrt{-\frac{B n^{2}}{2 A n-2 n-A}}\left(\eta+\eta_{0}\right)\right)\right)^{2}\right]^{1 / 2 n} \times\left(e^{\iota \Psi(x, t)}\right) .
$$

Set 6: If $\tau<0$ and $\mu=0$,

$$
\begin{array}{r}
u_{6}(x, t)=\left[F_{2}\left(\cos \left(\sqrt{\frac{B n^{2}}{2 A n-2 n-A}}\left(\eta+\eta_{0}\right)\right)+\iota \sin \left(\sqrt{\frac{B n^{2}}{2 A n-2 n-A}}\left(\eta+\eta_{0}\right)\right)\right)^{2}\right]^{1 / 2 n} \\
\times\left(e^{\iota \Psi(x, t)}\right) .
\end{array}
$$


Where $\Psi(x, t)=-k x+w t+\epsilon, \quad \eta=x-c t$.

\section{Form 2:}

Using balance principal on (11), attains $N=2$, so (12) gives

$$
W(\eta)=F_{0}+F_{1} \Phi(\eta)+F_{2}(\Phi(\eta))^{2},
$$

where $F_{0}, F_{1}$ and $F_{2}$ are constants. Inserting (37) in (11) and comparing the coefficients of each polynomial of $\Phi(\eta)$ to zero, we retrieve a set of equations in $F_{0}, F_{1}, F_{2}, \tau$ and $\mu$. Working on the set of equations, we acquire

$$
\begin{gathered}
F_{0}=\frac{(A(1-2 n)+3 n) B}{k(\theta A(1-2 n)+2 n \theta+\phi A(1-2 n)+2 n \phi)}, \\
F_{1}=0, \quad F_{2}=\frac{\mu^{2}(A(1-2 n)+3 n)}{k n^{2}(\phi+\theta)}, \\
\tau=-\frac{B n^{2}}{(2 n+A(1-2 n)) \mu}, \quad \mu=\mu .
\end{gathered}
$$

Set 1: If $\tau \mu>0$,

$$
\begin{array}{r}
u_{9}(x, t)=\left[\frac{(A(1-2 n)+3 n) B}{k(\theta A(1-2 n)+2 n \theta+\phi A(1-2 n)+2 n \phi)}\right. \\
\left.+F_{2}\left(\chi \sqrt{-\frac{B n^{2}}{(2 n+A(1-2 n)) \mu^{2}}} \tan \left(\sqrt{-\frac{B n^{2}}{A+2 n-2 A n}}\left(\eta+\eta_{0}\right)\right)\right)^{2}\right]^{1 / 2 n} \times\left(e^{\iota \Psi(x, t)}\right) .
\end{array}
$$

Set 2: If $\tau \mu>0$,

$$
\begin{array}{r}
u_{10}(x, t)=\left[\frac{(A(1-2 n)+3 n) B}{k(\theta A(1-2 n)+2 n \theta+\phi A(1-2 n)+2 n \phi)}\right. \\
\left.+F_{2}\left(\chi \sqrt{-\frac{B n^{2}}{(2 n+A(1-2 n)) \mu^{2}}} \cot \left(\sqrt{-\frac{B n^{2}}{A+2 n-2 A n}}\left(\eta+\eta_{0}\right)\right)\right)^{2}\right]^{1 / 2 n} \times\left(e^{\iota \Psi(x, t)}\right) .
\end{array}
$$

Set 3: If $\tau \mu<0$,

$$
\begin{array}{r}
u_{11}(x, t)=\left[\frac{(A(1-2 n)+3 n) B}{k(\theta A(1-2 n)+2 n \theta+\phi A(1-2 n)+2 n \phi)}\right. \\
\left.+F_{2}\left(\chi \sqrt{\frac{B n^{2}}{(2 n+A(1-2 n)) \mu^{2}}} \tanh \left(\sqrt{\frac{B n^{2}}{A+2 n-2 A n}}\left(\eta+\eta_{0}\right)\right)\right)^{2}\right]^{1 / 2 n} \times\left(e^{\iota \Psi(x, t)}\right) .
\end{array}
$$


Set 4: If $\tau \mu<0$,

$$
\begin{array}{r}
u_{12}(x, t)=\left[\frac{(A(1-2 n)+3 n) B}{k(\theta A(1-2 n)+2 n \theta+\phi A(1-2 n)+2 n \phi)}\right. \\
\left.+F_{2}\left(\chi \sqrt{\frac{B n^{2}}{(2 n+A(1-2 n)) \mu^{2}}} \operatorname{coth}\left(\sqrt{\frac{B n^{2}}{A+2 n-2 A n}}\left(\eta+\eta_{0}\right)\right)\right)^{2}\right]^{1 / 2 n} \times\left(e^{\iota \Psi(x, t)}\right) .
\end{array}
$$

where $\chi=\operatorname{sgn}\left(-\frac{B n^{2}}{(2 n+A(1-2 n)) \mu}\right), \quad \Psi(x, t)=-k x+w t+\epsilon, \quad \eta=x-c t$.

\section{$5 \quad$ Results and Discussions}

we illustrated the solutions of BAE in the form of optical solitons using new EHFM. These solutions have applications in telecommunication to transfer information as solitons have the proficiency to travel long spaces without distortion and without altering their shapes. In this work, we added selected graphical representations of some solutions of BAE to dodge overcrowded the manuscript. Figs. 1-6 represent 2-D and 3-D plots of some optical solitons of (1). Figures 1,6 demonstrate the solutions obtained given in (31) and (42) accordingly, which are singular soliton waves. Whereas figures 2,4 demonstrate the solutions (32) and (39) which are periodic singular soliton waves. Solution (33) given by Figure 3 represents bright soliton and solution (41) provide dark soliton and is shown by Figure 5 .

(a)

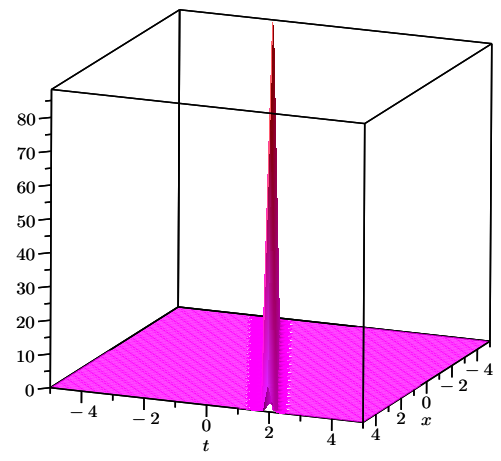

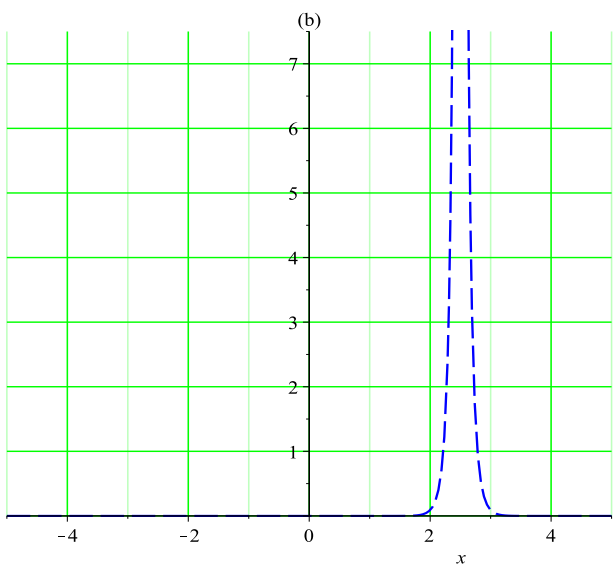

Figure 1: (a) 3D plot of (31) with $F_{2}=1.7, \quad A=-2.7, \quad B=11.4, \quad n=1 \quad k=$ $-2, \quad \theta=-4, \quad \phi=0.8, \quad \omega=3, c=2.5, \quad \epsilon=-4$.(b) $2 \mathrm{D}$ representation of $(31)$ using $t=1$. 

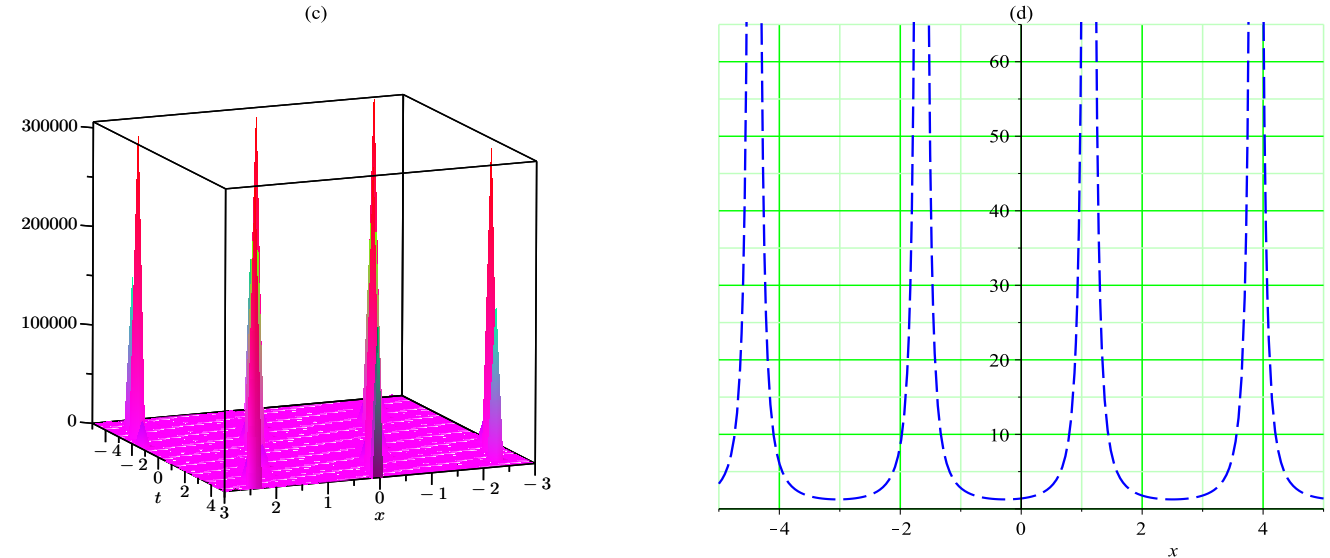

Figure 2: (c) 3D plot of (32) with $F_{2}=1.7, \quad A=-2.7, \quad B=11.4, \quad n=1 \quad k=$ $-2, \quad \theta=-4, \phi=0.8, \quad \omega=3, \epsilon=4, c=2.5$.(d) $2 \mathrm{D}$ representation of (32) with $t=1$.
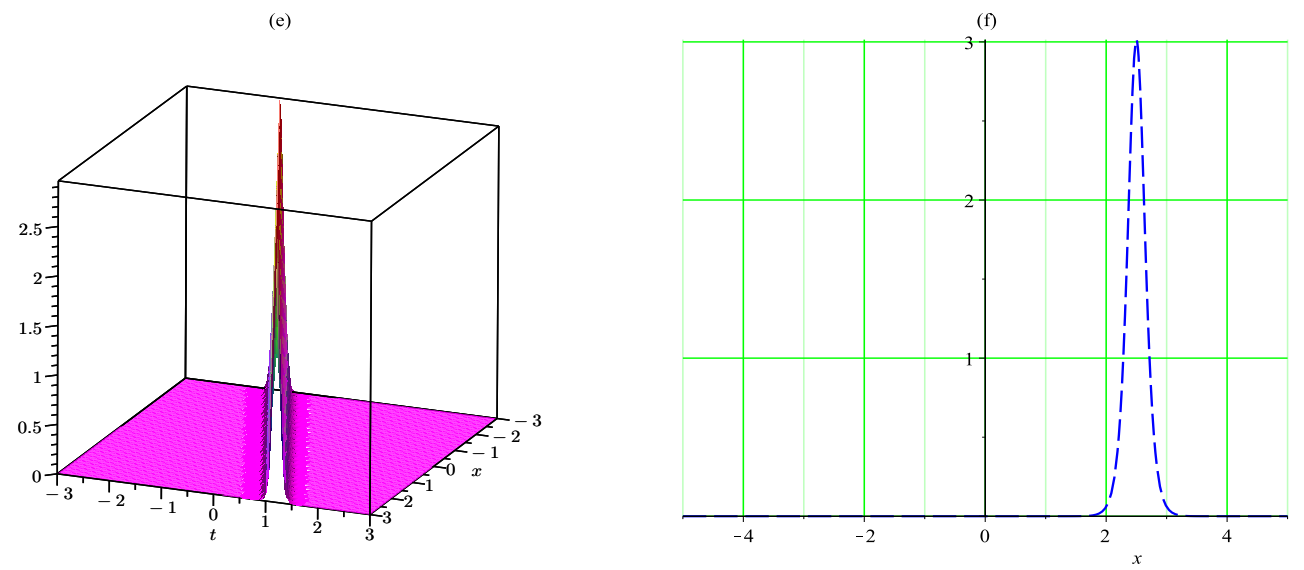

Figure 3: (e) 3D plot of (33) for $F_{2}=1.7, \quad A=-2.7, \quad B=11.4, \quad n=1 \quad k=$ $-2, \quad \theta=-4, \phi=0.8, \quad \omega=3, \epsilon=-4, c=2.5$.(f) $2 \mathrm{D}$ representation of (33) for $t=1$. 

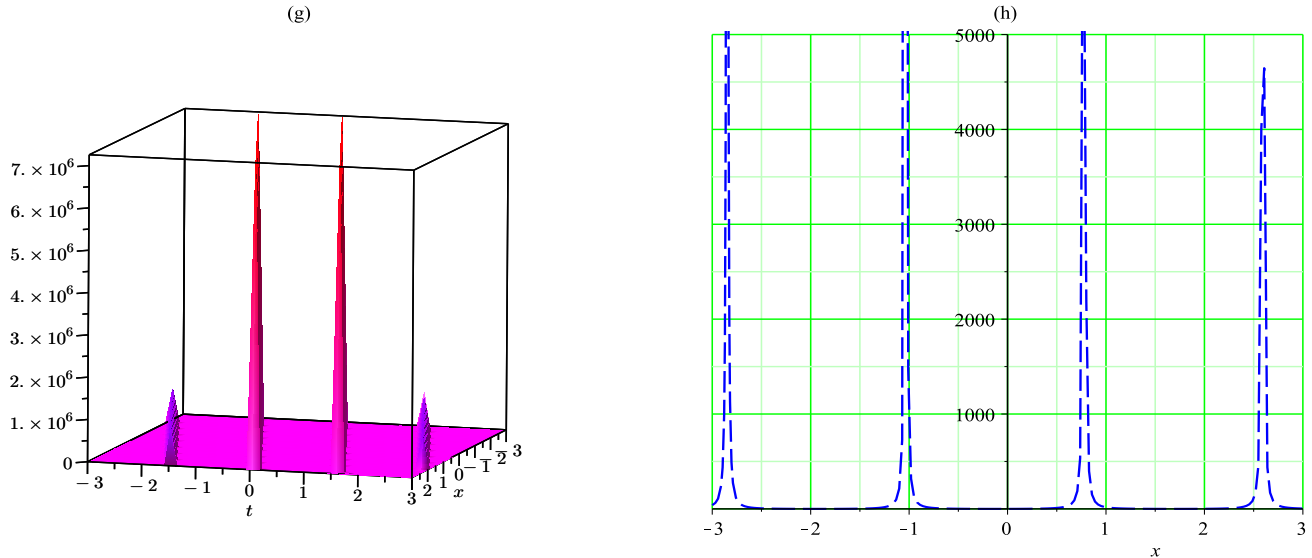

Figure 4: (g) 3D graph of (41) for $F_{2}=1.7, \quad A=-2.7, \quad B=11.4, \quad n=1 \quad k=$ $-2, \quad \theta=-4, \quad \phi=0.8, \quad \omega=3, \epsilon=-4, c=2.5$. (h) $2 \mathrm{D}$ representation of (41) for $t=1$.
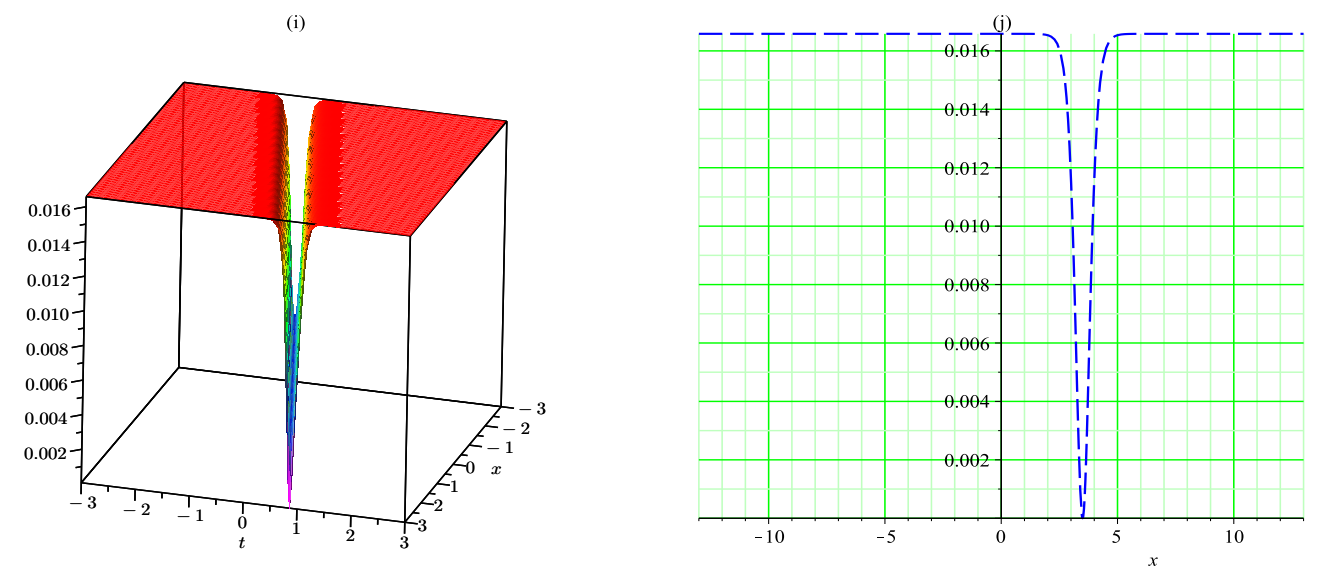

Figure 5: (i) 3D graph of (43) for $F_{2}=1.7, \quad A=-2.7, \quad B=11.4, n=1 \quad k=$ $-2, \quad \theta=-4, \phi=0.8, \quad \omega=3, \epsilon=-4, c=2.5$. (j) $2 \mathrm{D}$ representation of (43) for $t=1$. 

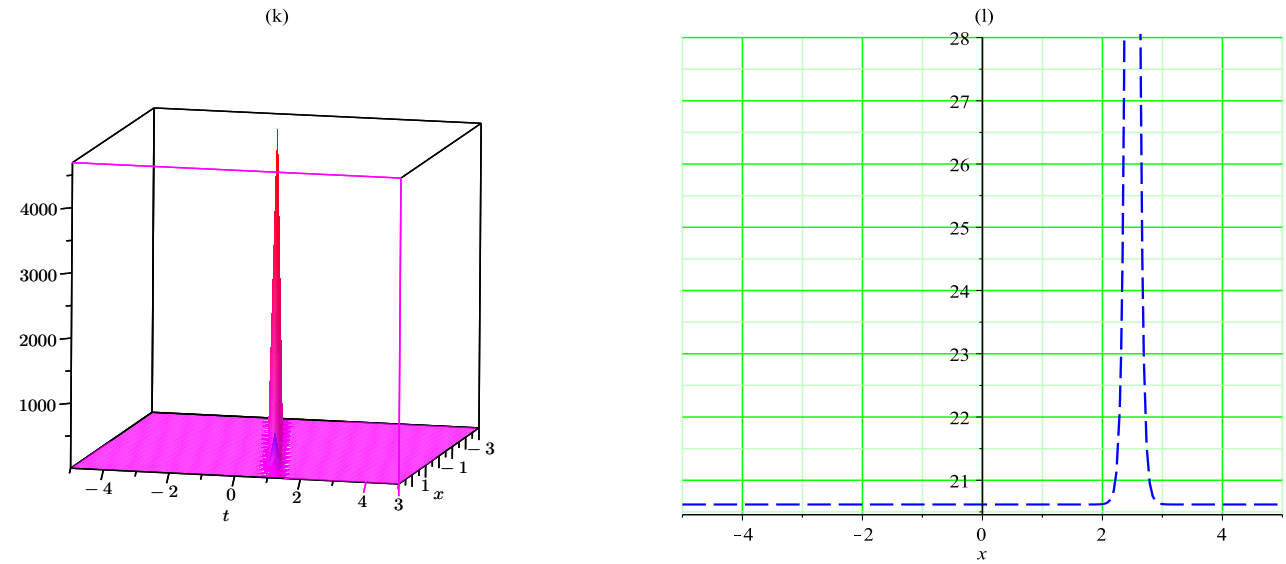

Figure 6: (k) 3D graph of (44) with $F_{2}=1.7, \quad A=-2.7, \quad B=11.4, n=1 \quad k=$ $-2, \theta=-4, \phi=0.8, \omega=3, \epsilon=-4, c=2.5$. (l) $2 \mathrm{D}$ representation of (44) for $t=1$.

\section{Conclusion}

In this paper, we have successfully employed the EHFM on BAE and constructed the bright, periodic, dark, singular and combo solitons. These constructed results have much importance in many areas of non-linear sciences such as physics, birefringent fibers, applied mathematics, optical fibers, engineering, pulse propagation and many more. The outcomes of this study are motivating and improve our understanding for the optical soliton solutions. By these outcomes, we can recognize that the present method is appropriate, useful and skilled for attaining the exact solutions of such kind of problems. The acquired results are fresh, correct and not reported earlier inliterature.

\section{References}

[1] A. Biswas, Optical soliton perturbation with Radhakrishnan-Kundu-Lakshmanan equation by traveling wave hypothesis, Optik, 171 (2018) 217-220.

[2] A. Biswas, A. J. M. Jawad, Q. Zhou, Resonant optical solitons with anti-cubic nonlinearity, Optik, 157 (2018) 525-31.

[3] S. Arshed, Two reliable techniques for the soliton solutions of perturbed GerdjikovIvanov equation, Optik, 164 (2018) 93-99.

[4] M. S. Osman, Nonlinear interaction of solitary waves described by multi-rational wave solutions of the $(2+1)$-dimensional Kadomtsev-Petviashvili equation with variable coefficients, Nonlinear Dynamics, 87 (2017) 1209-16. 
[5] F. Tahir, M. Younis, H. U. Rehman, Optical Gaussons and dark solitons in directional couplers with spatiotemporal dispersion, Optical and Quantum Electronics, 49 (2017) 422.

[6] A. Zafar, Rational exponential solutions of conformable space-time fractional equal width equations Nonlinear Engineering, 8 (2019) 350-5.

[7] Y. Ren, H. Zhang, New generalized hyperbolic functions and auto-Backlund transformations to find new exact solutions of the $(2+1)$-dimensional NNV equation, physics Letter A, 357 (6)(2006) 438-448.

[8] H. Rezazadeh, S. M. Mirhosseini-Alizamini, M. Eslami, M. Rezazadeh, M. Mirzazadeh, S. Abbagari, New optical solitons of nonlinear conformable fractional Schrodinger-Hirota equation, Optik, 172 (2018) 545-553.

[9] M. Quiroga-Teixeiro, H. Michinel, Stable azimuthal stationary state in quintic nonlinear optical media, J. Opt. Soc. Amer. B, 14 (1997) 2004-2009.

[10] H. U. Rehman, N. Ullah, M. A. Imran, Highly dispersive optical solitons using Kudryashov's method, Optik, 199 (2019) 163349.

[11] M. Ekici, M. Mirzazadeh, A. Sonmezoglu, M. Z. Ullah, M. Asma, Q. Zhou, S.P. Moshokoa, A. Biswas, M. Belic, Optical solitons with Schrodinger-Hirota equation by extended trial equation method, Optik, 136 (2017) 451-461.

[12] J. Manaflan, M. Lakestani, Optical soliton solutions for the Gerdjikov-Ivanov model via tan ( $\phi / 2)$-expansion method, Optik, 127 (20) (2016) 9603-9620.

[13] D. Lu, K. U. Tariq, M. S. D. Osman, M. Younis, M. M. A. Khater, New analytical wave structures for the $(3+1)$-dimensional Kadomtsev-Petviashvili and the generalized Boussinesq models and their applications, Results in Physics, 14 (2019) 102491.

[14] Q. Zhou, M. Ekici, A. Sonmezoglu, Exact chirped singular soliton solutions of TrikiBiswas equation, Optik, 181 (2019) 338-342

[15] X. Liu, H. Triki, Q. Zhou, M. Mirzazadeh, W. Liu, A. Biswas, M. Belic, Generation and control of multiple solitons under the influence of parameters, Nonlinear Dynamics, 95 (2019) 143-150.

[16] W. Yu, Q. Zhou, M. Mirzazadeh, W. Liu, A. Biswas, Phase shift, ampliflcation, oscillation and attenuation of solitons in nonlinear optics, J. Adv. Res. 15 (2019) 69-76. 
[17] A. Bansal, A. H. Kara, A. Biswas, S. Khan, Q. Zhou, S.P. Moshokoae, Optical solitons and conservation laws with polarization-mode dispersion for coupled FokasLenells equation using group invariance, Chaos, Solitons and Fractals, 120 (2019) 245-249.

[18] H. U. Rehman, M. S. Saleem, M. Zubair, S. Jafar, I. Latif, Optical solitons with Biswas-Arshed model using mapping method, Optik, (2019) 163091.

[19] M. Tahir, A. U. Awan, H. U. Rehman, Optical solitons to Kundu-Eckhaus equation in birefringent flbers without four-wave mixing, Optik, 199 (2019) 163297.

[20] N. Ullah, H. Rehman, M. A. Imran, T. Abdeljawad, Highly dispersive optical solitons with cubic law and cubic-quintic-septic law nonlinearities, Results in Physics, 17 (2020) 103021.

[21] A. U. Awan, M. Tahir, H. U. Rehman, On traveling wave solutions: The Wu-Zhang system describing dispersive long waves, Modern Physics Letters B, 33(6) (2019) 1950059.

[22] X. Liu, H. Triki, Q. Zhou, W. Liu, A. Biswas, Analytic study on interactions between periodic solitons with controllable parameters, Nonlinear Dynamics, 94 (1) (2018) 703-709.

[23] A. Messouber, H. Triki, F. Azzouzi, Q. Zhou, A. Biswas, S. P. Moshokoa, M. Belic, Propagation properties of dipole-managed solitons through an inhomogeneous cubicquintic-septic medium, Optics Communications, 425 (2018) 64-70.

[24] A. Biswas, Q. Zhou, H. Triki, M. Z. Ullah, M. Asma, S.P. Moshokoa, Resonant optical solitons with parabolic and dual-power laws by semi-inverse variational principle, Journal of Modern Optics, 65 (2) (2018) 179-184.

[25] R. W. Kohl, A. Biswas, M. Ekici, S. Khan, A. S. Alshomrani, Belic M R Alshomrani, Highly dispersive optical soliton perturbation with kerr law by semi-inverse variational principle, Optik, 199 (2019) 163226.

[26] A. Seadawy, D. Lu, Bright and dark solitary wave soliton solutions for the generalized higher order nonlinear Schrödinger's equation and its stability, Results in Physics, 7 (2017) 43.

[27] H. Triki, H. Hayat, O. M. Aldossary, A. Biswas, Bright and dark solitons for the resonant nonlinear Schrodinger's equation with time-dependent coefficients, Opt. Laser Technol. 44 (2012) 2223-31.

[28] M. Saha, A. K. Sarma, A. Biswas, Dark optical solitons in power law media with time-dependent coefficients, Phys. Lett. A, 373 (2009) 4438-41. 
[29] M. S. Osman, A. M. Wazwaz, A general bilinear form to generate different wave structures of solitons for a $(3+1)$ - dimensional Boiti-Leon-Manna-Pempinelli equation, Math. Methods Appl. Sci., 42 (2019) 6277-83.

[30] Y. Ding, M. S. Osman, A. M. Wazwaz, Abundant complex wave solutions for the nonautonomous Fokas-Lenells equation in presence of perturbation terms, Optik, 181 (2019) 503-13.

[31] J. G. Liu, M. S. Osman, A. M. Wazwaz, A variety of nonautonomous complex wave solutions for the $(2+1)$ - dimensional nonlinear Schrodinger equation with variable coefficients in nonlinear optical fibers, Optik, 180 (2019) 917-23.

[32] S. Arshed, A. Biswas, M. Abdelaty, Q. Zhou, S.P. Moshokoa, M. Belic, Optical soliton perturbation for Gerdjikov-Ivanov equation via two analytical techniques, Chinese Journal of Physics, 56 (6) (2018) 2879-2886.

[33] A. Biswas, M. Ekici, A. Sonmezoglu, R. T. Alqahtani, Sub-pico-second chirped optical solitons in mono-mode flbers with Kaup-Newell equation by extended trial function method, Optik, 168 (2018) 208-216.

[34] K. K. Ali, R. I. Nuruddeen, K. R. Raslan, New structures for the space-time fractional simplified MCH and SRLW equations, Chaos, Solitons and Fractals, 106 (2018) 304-9.

[35] H. U. Rehman, N. Ullah, M. A. Imran, Optical solitons of Biswas-Arshed equation in birefringent fibers using extended direct algebraic method, Optic, (2020) 165378.

[36] A. Biswas, S. Arshed, Optical solitons in presence of higher order dispersions and absence of self-phase modulation, Optik, 174 (2018) 452-459.

[37] Hamood Ur Rehman, Sobia Jafar, Ahmad Javed, Safdar Hussain, Muhammad Tahir, New optical soliotons of Biswas-Arshed equation using different technique, Optik, 206 (2020) 163670.

[38] Muhammad Tahir, Aziz Ullah Awan, Hamood ur Rehman, Dark and singular optical solitons to the Biswas-Arshed model with Kerr and power law nonlinearity, Optik, 185 (2019) 777-783.

[39] Prakash Kumar Das, Chirped and chirp-free optical exact solutions of the BiswasArshed equation with full nonlinearity by the rapidly convergent approximation method, Optic, 223 (2020) 165293. 
[40] Zeliha Korpinar, Mustafa Inc, Mustafa Bayram, Mir Sajjad, Hashemi, New optical solitons for Biswas-Arshed equation with higher order dispersions and full nonlinearity, Optic, 206 (2020) 163332.

[41] Muhammad Tahir, Aziz Ullah Awan, Optical singular and dark solitons with Biswas-Arshed model by modified simple equation method, Optic, 202 (2020) 163523.

[42] Mehmet Ekici, Abdullah Sonmezoglu, Optical solitons with Biswas-Arshed equation by extended trial function method, Optic, 177 (2019) 13-20.

[43] Yadong Shang, The extended hyperbolic function method and exact solutions of the long-short wave resonance equations, Chaos, Solitons and Fractals, 363 (2008) 762-771.

[44] The extended hyperbolic functions method and new exact solutions to the Zakharov equations, Applied Mathematics and Computation, 2001 (2008) 110-122.

[45] S. Nestor, A. Houwe, G. Betchewe, M. Inc, and S. Y. Doka, A series of abundant new optical solitons to the conformable space-time fractional perturbed nonlinear Schrödinger equation, Physica Scripta, (2020). 